\title{
A Preliminary Study of the Association between SOX17 Gene Variants and Intracranial Aneurysms Using Exome Sequencing
}

\author{
Jeong Jin Park, ${ }^{1}$ Bong Jun Kim, ${ }^{2}$ Dong Hyuk Youn, ${ }^{2}$ Hyuk Jai Choi, ${ }^{3}$ Jin Pyeong Jeon ${ }^{2,3}$ \\ Department of Neurology, Konkuk University Medical Center, Seoul, Korea \\ Institute of New Frontier Research, ${ }^{2}$ Hallym University College of Medicine, Chuncheon, Korea \\ Department of Neurosurgery, ${ }^{3}$ Hallym University College of Medicine, Chuncheon, Korea
}

Objective : Conflicting results regarding SOX17 genes and the risk of intracranial aneurysms (IA) exist in the Korean population, although significant positive correlations were noted in genome-wide association studies in European and Japanese populations. Therefore, we aimed to investigate an association between SOX17 gene variants and IA using exome sequencing data.

Methods : This study included 26 age-gender matched IA patients and 26 control subjects. The SOX17 gene variants identified from whole-exome sequencing data were examined. Genetic associations to estimate odds ratio (OR) and $95 \%$ confidence interval (Cl) were performed using the software EPACTS.

Results : The mean age of the IA and control groups were $51.0 \pm 9.3$ years and $49.4 \pm 14.3$ years, respectively ( $p=0.623$ ). Seven variants of $50 \times 17$, including six single nucleotide polymorphisms and one insertion and deletion, were observed. Among these variants, rs12544958 $(A>G)$ showed the most association with IA, but the association was not statistically significant (OR, 1.97; $95 \%$ $\mathrm{Cl}, 0.81-4.74 ; p=0.125$ ). Minor allele frequencies of the IA patients and controls were 0.788 and 0.653 , respectively. None of the remaining variants were significantly associated with IA formation.

Conclusion : No significant association between SOX17 gene variants and IA were noted in the Korean population. A large-scale exome sequencing study is necessary to investigate any Korean-specific genetic susceptibility to IA.

Key Words : Intracranial aneurysm · Gene · Whole exome sequencing.

\section{INTRODUCTION}

Intracranial aneurysm (IA) refer to ballooning or bulging of the intracranial arteries, causing life-threatening subarachnoid hemorrhage (SAH) with annual rupture rate up to
$2 \%{ }^{1,3,12)}$. Although the exact mechanisms of IA formation remain unclear, clinical risk factors and genetic susceptibility are known to contribute to IA formation and growth ${ }^{3}$. Wellknown clinical risk factors for IA are female gender, cigarette smoking, hypertension (HTN), and familial aneurysm ${ }^{22}$.

- Received : November 7, 2019 •Revised : January 22, 2020 •Accepted : February 12, 2020

- Address for reprints : Jin Pyeong Jeon

Department of Neurosurgery, Hallym University College of Medicine, 77 Sakju-ro, Chuncheon 24253, Korea

Tel : +82-33-240-5171, Fax : +82-33-240-9970, E-mail : jjs6553@daum.net, ORCID : https://orcid.org/0000-0001-8543-6855

This is an Open Access article distributed under the terms of the Creative Commons Attribution Non-Commercial License (http://creativecommons.org/licenses/by-nc/4.0) which permits unrestricted non-commercial use, distribution, and reproduction in any medium, provided the original work is properly cited. 
Candidate genes identified from meta-analysis using genomewide association studies (GWASs) were SRY-box 17 (SOX17, rs9298506, rs10958409), endothelin receptor A (rs6841581), and cyclin-dependent kinase inhibitor $2 \mathrm{~B}$ antisense inhibitor (rs10757278 and rs1333040) genes in European and Japanese populations $^{1)}$. Among those candidate genes, SOX17 has been studied in the Korean population. Hong et al. ${ }^{13)}$ reported a protective effect of the minor $\mathrm{C}$ allele of rs1072737 on IA formation (odds ratio [OR], 0.69; 95\% confidence interval [CI], 0.49-0.96). Other single nucleotide polymorphisms (SNPs), such as rs9298506, rs10958409, and rs1504749, were not associated with IA. These conflicting results suggested the effect of different SOX17 gene variants on IA formation according to inter-and intra-ethnic population differences.

Genomic data have been accumulated in the current precision medicine areas ${ }^{7)}$. Beyond GWAS, next generation sequencing (NGS) has been widely performed since the mid2000s. Whole-exome sequencing (WES) allows informations about genetic and protein-coding variants, particularly in selected regions of interest ${ }^{23,25)}$. In this study, we aimed to firstly report the association between SOX17 gene variants and IA using exome sequencing data in the Korean population.

\section{MATERIALS AND METHODS}

\section{Study population}

This study was approved by the Institutional Review Boards of the Chuncheon Sacred Heart Hospital (No. 2017-9 and 2018-6). This study included almost age-gender matched IA patients and control subjects who visited the participating institution between April 2018 and June 2019 ${ }^{17)}$. The inclusion criteria of the study were 1) adult patients over 18 years of age with 2) sporadic aneurysms with saccular shapes. The exclusion criteria were 1) fusiform, dissected, traumatic or infectious aneurysms and 2) patients with concomitant other cerebrovascular diseases. Patients who met the following criteria were selected as controls : 1) patients who underwent radiologic tests for headache or medical check-ups with 2) no familial history of IA or SAH among the first-degree relatives $^{6,9,12,13,18)}$.

The primary outcome of the study was to evaluate the associations between SOX17 gene variants and IA using exome sequencing data to identify a potential marker for IA formation.
Patient medical records were reviewed to collect clinical and angiographic characteristics (sex, age, HTN, diabetes mellitus (DM), hyperlipidemia, smoking history ${ }^{15)}$, initial clinical presentation, and aneurysm size and location (anterior. vs. posterior circulation) $)^{5}$.

\section{Gene sequencing}

Genomic DNA was isolated from peripheral blood cells with the QIAamp DNA Blood Midi Kit (QIAGEN, Hilden, Germany) ${ }^{13)}$. WES was performed using a NovaSeq Sequencer (Illumnia, Inc., San Diego, CA, USA) following the manufacturer's protocols. The read depths of the IA and control were $>200 \times$ and $>100 \times$, respectively, with an average sequence coverage over the targeted region of $>90 \%{ }^{8)}$. The targeted gene, SOX17, was selected from previous studies that reported an association with the pathogenesis of IA development ${ }^{13,20)}$. Finally, we detected 1432 variants containing seven heterozygotes and 1425 homozygotes. Quality control was provided by using the Genome Analysis Toolkit (GATK) best practice workflow (gatk-4.0.6.0). Sequence reads were aligned to the reference sequence (hs37.fa) with BWA mem (bwa 0.7.17). Conversion of the SAM file to a binary file was accomplished using samtools (0.1.19-44428cd). Picard (picard-2.18.9) was used for various implementations such as sorting and inputting of sample information and removal of duplicates. Base quality score recalibration and variant quality reacaribration were performed. Genetic variants were identified using haplotypecaller with a genomic varinat call file.

\section{Statistical analyses}

Continuous variables are presented as mean \pm standard deviation. Univariate analyses by Student's t-tests and Pearson's chi-squared tests were performed to evaluate the differences between the two groups using MedCalc software (Medcalc, Mariakerke, Belgium). Genetic association and visualization of the results (Manhattan plot and quantile-quantile plot [Q-Q plot]) were analyzed using the software EPACTS (Efficient and Parallelizable Association Container Toolbox; http:// genome.sph.umich.edu/wiki/EPACTS) under a linear mixed model, estimating the OR with 95\% CI. A web-based tool, LocusZoom (http://locuszoom.org), was used to focus on the location of the SOX17 gene variants. 


\section{RESULTS}

\section{Demographic characteristics of the study cohorts}

The baseline characteristics of 26 IA patients and 26 healthy controls are described in Table 1. The mean age of the two groups was $49.4 \pm 14.3$ in the control subjects and $51.0 \pm 9.3$ in the IA patients ( $p=0.623$ ). More IA patients appeared to have HTN than the control subjects, but the difference was not statistically significant (IA, $\mathrm{n}=8$ [30.8\%] vs. control, $\mathrm{n}=3$ [11.5\%]) ( $p=0.093$ ). Other variables, such as female gender, DM, hyperlipidemia, and smoking, did not differ significantly between the two groups. SAH presentation was noted in 15 (57.7\%) out of 26 IA patients. There were $21(80.8 \%)$ anterior circulation aneurysms, five internal carotid artery aneurysms, five in the

Table 1. Baseline characteristics of patients harboring IA and controls

\begin{tabular}{lccc}
\hline Variable & IA (n=26) & $\begin{array}{c}\text { Controls } \\
(\mathbf{n = 2 6 )}\end{array}$ & $\boldsymbol{p}$-value* \\
\hline Female & $18(69.2)$ & $13(50.0)$ & 0.162 \\
Age (years) & $51.0 \pm 9.3$ & $49.4 \pm 14.3$ & 0.623 \\
Hypertension & $8(30.8)$ & $3(11.5)$ & 0.093 \\
Diabetes mellitus & $1(3.8)$ & $1(3.8)$ & 1.000 \\
Hyperlipidemia & $3(11.5)$ & $1(3.8)$ & 0.301 \\
Cigarette smoking & $2(7.7)$ & $3(11.5)$ & 0.641 \\
SAH presentation & $15(57.7)$ & &
\end{tabular}

Values are presented as mean \pm standard deviation or number (\%). ${ }^{*} p<0.05$ is significant. IA : intracranial aneurysms, SAH : subarachnoid hemorrhage anterior communicating artery or anterior cerebral artery, seven in the middle cerebral artery, and four in the posterior communicating artery.

\section{Associations of SOX17 gene variants with IA}

The Manhattans plots showed seven variants located in the

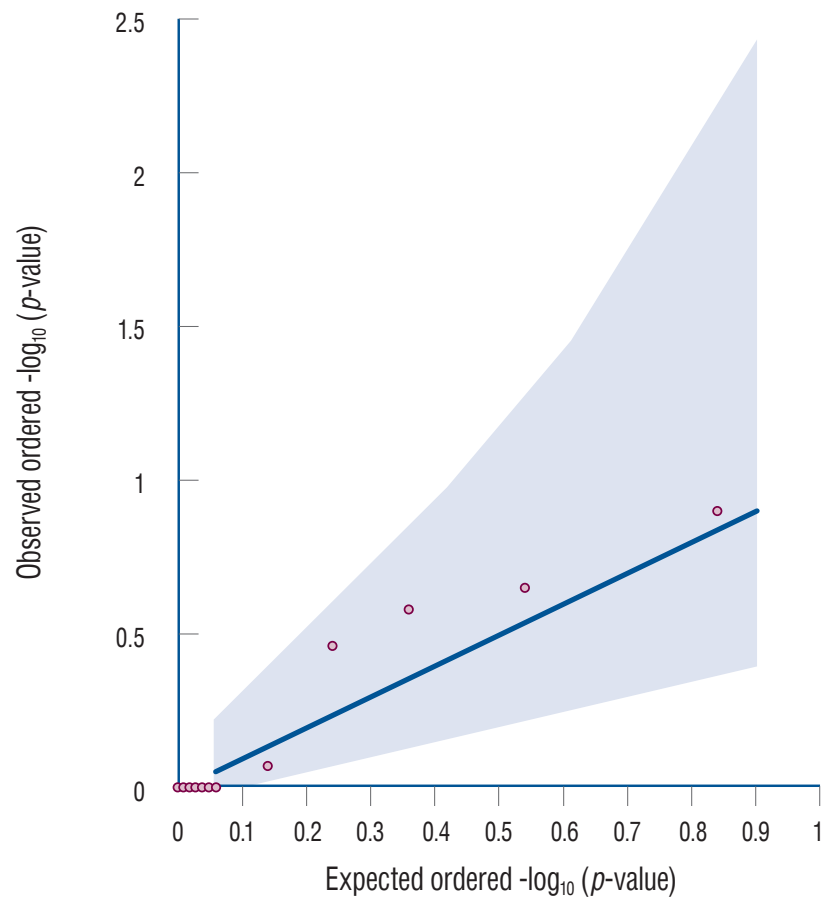

Fig. 2. Quantile-quantile plot for single variant analysis of the SOX17 gene. This plot shows observed versus expected ordered $-\log _{10}$ ( $p$-values). The shaded regions indicates standard errors ${ }^{10)}$.

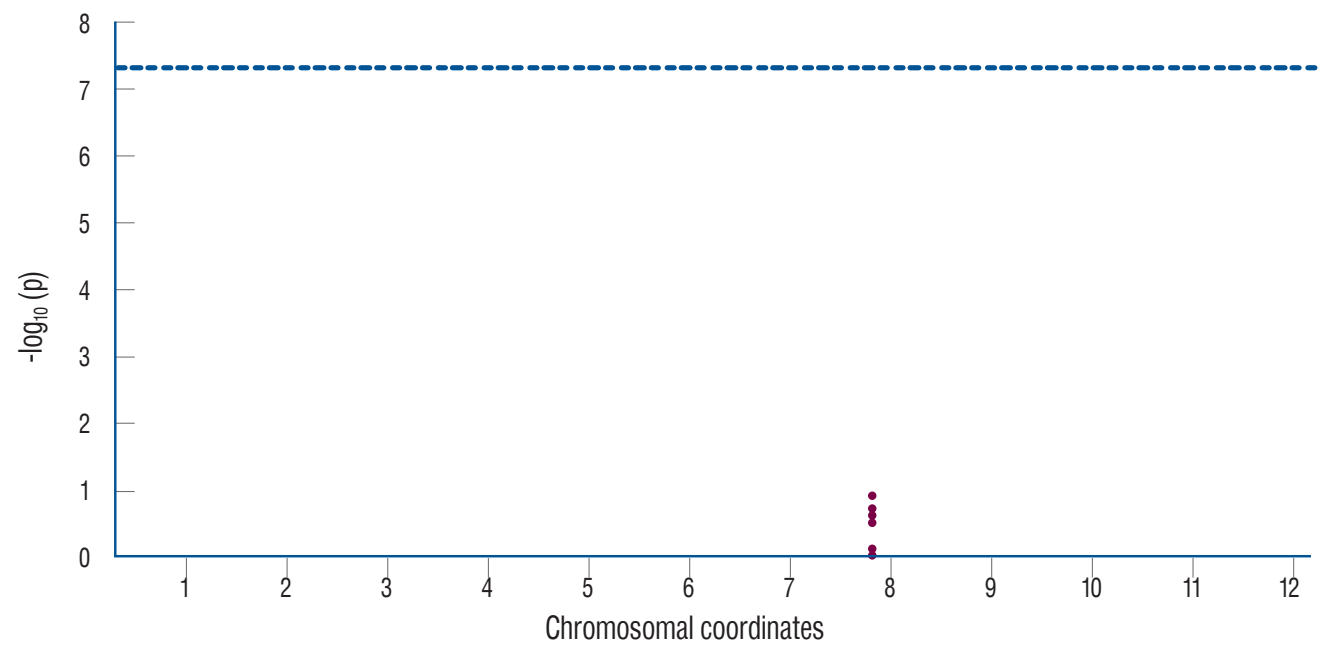

Fig. 1. The Manhattan plot for single variant analysis of the SOX17 gene (shown in red dots) in 26 patients with intracranial aneurysms and 26 control subjects. The gray dotted line indicates statistical significance after correction for multiple testing. 
SOX17 gene, but none of these variants passed the genomewide significant level (Fig. 1). The Q-Q plot for single variant analysis of the SOX17 gene showed that all variants were distributed in the gray area, indicating no meaningful association between SOX17 gene variants and IA (Fig. 2).

A total of seven variants of SOX17, six SNPs and one insertion and deletion, were observed in this study. Among them, rs12544958 (A>G) appeared to be associated with IA, but was not statistically significant (OR, 1.97; 95\% CI, 0.81-4.74; $p=0.125$ ). More specifically, the minor allele frequency of the
IA and controls were 0.788 and 0.653 , respectively. Two SNPs of rs12545485 (T>C) and rs73679444 (C>A), which were located within $6 \mathrm{bp}$, also did not have a meaningful association with IA. There were the same number of minor rs62516525 $(\mathrm{C}>\mathrm{T})$ alleles in both the IA and control groups (Table 2). We further constructed regional plots of rs12544958, which had the lowest $p$-value in our analysis. In the plot, the rs 12544958 variant described in the purple diamond shows a non-significant association with IA using genome sequencing (Fig. 3).

Table 2. Single variant analysis of the SOX17 gene between intracranial aneurysm patients and control subjects

\begin{tabular}{|c|c|c|c|c|c|c|c|}
\hline Chr. & Position & SNP or INDEL & MAF*, case/control & OR $(95 \% \mathrm{Cl})$ & $p$-value & Score & rsID \\
\hline 8 & 55371538 & $A / G$ & $0.788 / 0.653$ & $1.97(0.81-4.74)$ & 0.125 & 1.534 & rs12544958 \\
\hline 8 & 55372660 & $T / G$ & 0.096/0.038 & $2.66(0.49-14.38)$ & 0.223 & 1.219 & rs117273864 \\
\hline 8 & 55371478 & $\mathrm{~T} / \mathrm{C}$ & $0.750 / 0.635$ & $1.73(0.74-4.02)$ & 0.259 & 1.128 & rs12545485 \\
\hline 8 & 55371472 & $C / A$ & $0.365 / 0.269$ & $1.56(0.68-3.59)$ & 0.345 & 0.944 & rs73679444 \\
\hline 8 & 55371154 & $\mathrm{C} / \mathrm{T}$ & $0.385 / 0.404$ & $0.92(0.42-2.03)$ & 0.840 & -0.201 & rs12541742 \\
\hline 8 & 55370619 & $\mathrm{C} / \mathrm{T}$ & $0.385 / 0.385$ & $1(0.45-2.20)$ & 1 & 7.07E-16 & rs62516525 \\
\hline 8 & 55371080 & C/CAG Insertion & $0.385 / 0.385$ & $1(0.45-2.20)$ & 1 & 7.07E-16 & rs71554162 \\
\hline
\end{tabular}

*MAF in patients (intracranial aneurysm) and controls (right) groups. Chr. : chromosom, SNP : single nucleotide polymorphism, INDEL : insertion and deletion, MAF : minor allele frequency, $\mathrm{OR}$ : odds ratio, $\mathrm{Cl}$ : confidence interval

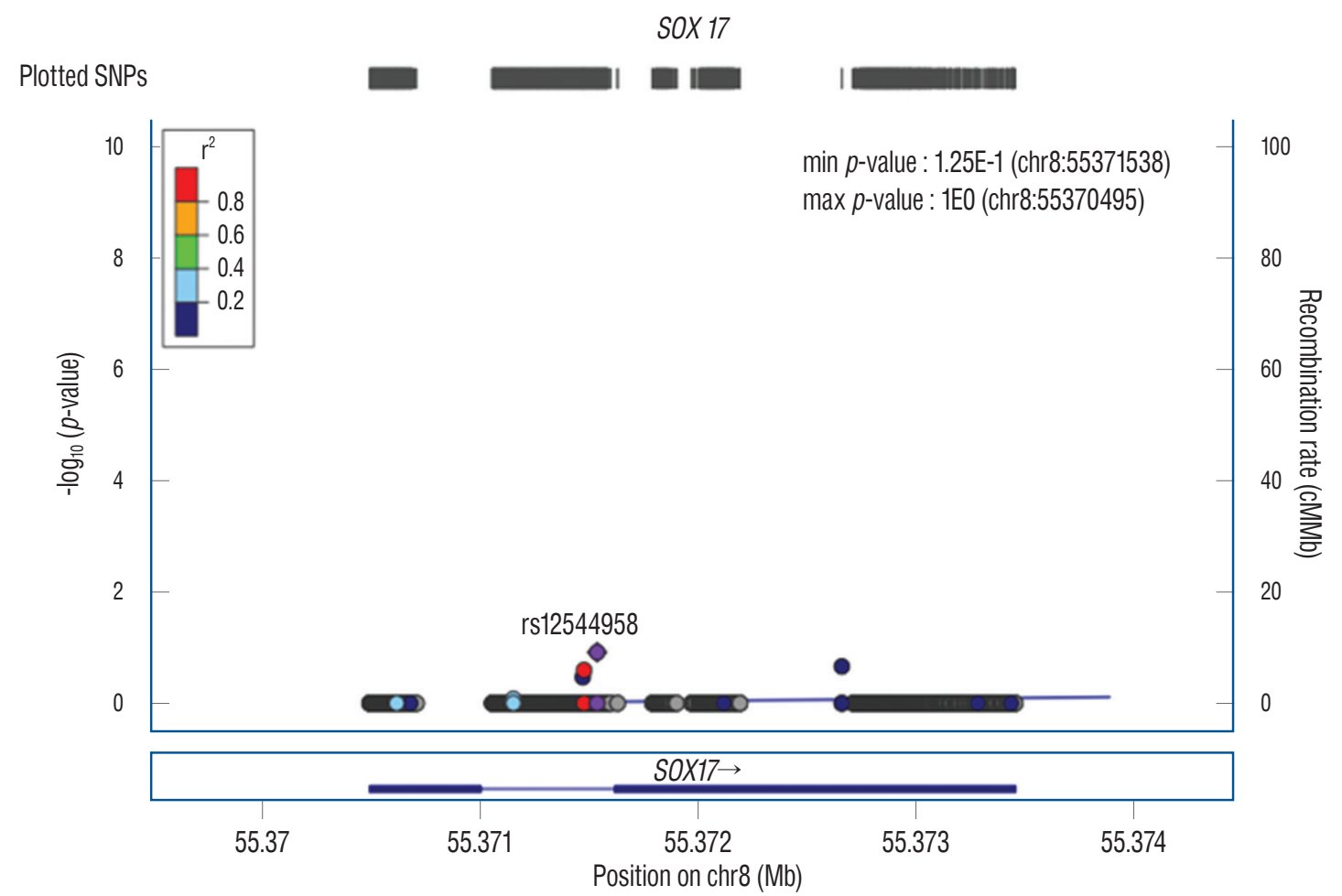

Fig. 3. A regional plot of the rs 12544958 variant's position $\pm 500 \mathrm{~kb}$ with IA development. The purple diamond indicates a non-significant association of the rs12544958 variant of the SOX17 gene with IA. SNP : single nucleotide polymorphism, IA : intracranial aneurysm. 


\section{DISCUSSION}

To the best of our knowledge, this was the first investigation examining SOX17 gene variants and IA using gene sequencing in the Korean population. The present study showed that SOX17 gene variants cannot be used as a reliable biological marker for Korean IA patients.

IA formation and growth has been related to endothelial changes, such as partial cell loss and intimal swelling, as well as medial layer damages ${ }^{4)}$. The SOX17 gene is robustly expressed in endothelial cells of the cerebral arteries ${ }^{20)}$. Lee et al. ${ }^{20)}$ demonstrated that vascular abnormalities mimicked IA formation in SOX17-deficient mouse with angiotensin II infusions, indicating SOX17 deficiency as a marker for IA formation. In their study, SOX17-deficient arteries had decreased platelet-derived growth factors, suggesting a potential impairment of paracrine regulation by SOX17 deficiency. Regarding humans, Alg et al. ${ }^{1)}$ reported that SOX17 gene variants increased IA risk, reporting an OR, 1.21 (95\% CI, 1.15-1.27) for rs9298506 and an OR, 1.19 (95\% CI, 1.13-1.26) for rs10958409. These associations were more prominent in the Caucasian population than in some Japanese cohorts, with an OR, 1.35 (95\% CI, 1.27-1.44) for rs9298506 and an OR, 1.31 (95\% CI, 1.23-1.39) for rs10958409 $9^{2,9,28)}$. In the Korean population, two SNPs of SOX17, rs9298506 and rs10958409, did not increase the IA risk (rs9298506 : OR, 1.06; 95\% CI, 0.781.43; and rs10958409 : OR, 1.01; 95\% CI, 0.75-1.35) ${ }^{13)}$. A GWAS also did not demonstrate an increased IA risk in Koreans harboring rs $9298506^{12)}$. In a Chinese population, no significant association was also observed (rs1504749 : OR, 1.05; 95\% CI, 0.821.35; and rs10958409: OR, 0.94; 95\% CI, 0.73-1.21) $)^{21)}$.

Recently, genetic variants associated with IA susceptibility identified by WES has been studied. Wu et al. ${ }^{26)}$ reported that c.C133T (p.H45Y) located within exon 2 of LOXL2 gene was associated with three familial IA patients. In addition, that association was also replicated in 28 individuals. Yamada et al. ${ }^{27}$ suggested susceptible loci of FAM160A1 and OR52E4 for SAH due to aneurysm rupture. In our study, seven SOX17 gene variants were not significantly associated with sporadic IAs. Nevertheless, different characteristics of the enrolled patients (sporadic vs. familial IAs) and genetic differences according to ethnicities should be considered when interpreting the data.

Korean genetic studies have focused on SNPs involved in endothelial dysfunction or extracellular matrix proteins. Song et al. $^{24)}$ reported that an endothelial nitric oxide synthase gene
(eNOS) T-786C mutation was not associated with IA size or IA rupture. Kim et al. ${ }^{19)}$ also did not find three IA-associated eNOS polymorphisms, 27VNTR, T786C, and G894T. Regarding the elastin gene, patients harboring the T allele of rs 2856728 demonstrated a statistically significant association with IA, particularly in males (OR, 3.45 in males vs. 1.88 in females) ${ }^{16)}$. Among the lysyl oxidase SNPs, rs2303656, rs3900446, and rs763497 increased IA risk significantly ${ }^{11)}$. A recent GWAS revealed eight new susceptibility loci, including GBA, TCF24, OLFML2A, ARHGAP32, CD163L1, CUL4A, LOC102724084, and $L R R C 3$, that had statistical power over 0.8 in Korean IA patients $^{12)}$. Only two SNPs, rs700651 and rs6841581, among the IA-susceptible SNPs identified in previous reports were validated in the Korean GWAS. Accordingly, further research employing the NGS platform is required to find Korean-specific IA loci that affect the susceptibility to IA formation and subsequent rupture.

This study had some limitations. The results may be underpowered to detect casual variations conferring susceptibility to IA due to the small number of enrolled patients ${ }^{13,14)}$. Although GATK is considered the gold standard for genomic analysis, the detection of variants depends on the references and quality controls that are applied in WES. Therefore, alternative analytic methods for prioritization of genetic variants, including additional variant-calling pipelines and integrative analyses of the genetic and environmental factors, are needed in the upcoming studies in a large number of sporadic IA patients. Nevertheless, this was the first genetic association study of SOX17 gene variants with IA from the WES database in the Korean population and it should be acknowledged.

\section{CONCLUSION}

No significant association between SOX17 gene variants and IA were observed in Korean IA patients. To investigate Korean-specific genetic susceptibility to IA, a large-scale exome sequencing data study is required.

\section{CONFLICTS OF INTEREST}

No potential conflict of interest relevant to this article was reported. 


\section{INFORMED CONSENT}

Informed consent was obtained from all individual participants included in this study.

\section{AUTHOR CONTRIBUTIONS}

\author{
Conceptualization : JPJ \\ Data curation : JJP, BJK, HJC \\ Formal analysis : BJK, DHY \\ Funding acquisition : JPJ \\ Methodology : BJK, JPJ \\ Project administration : JPJ \\ Visualization : BJK \\ Writing - original draft : JJP, BJK \\ Writing - review \& editing : JPJ
}

\section{ORCID}
Jeong Jin Park
https://orcid.org/0000-0001-9325-164X
Bong Jun Kim
https://orcid.org/0000-0003-3374-5554
Dong Hyuk Youn
https://orcid.org/0000-0003-2259-1844
Hyuk Jai Choi
https://orcid.org/0000-0002-3774-5941
Jin Pyeong Jeon

\section{- Acknowledgements}

This study was supported by the National Research Foundation of Korea funded by the Ministry of Science, Information and Communication Technologies and Future Planning of the Korea Government (Grant Number 2017R1C1B5016678).

\section{References}

1. Alg VS, Sofat R, Houlden $H$, Werring DJ : Genetic risk factors for intracranial aneurysms: a meta-analysis in more than 116,000 individuals. Neurology 80 : 2154-2165, 2013

2. Bilguvar $K$, Yasuno $K$, Niemelä $M$, Ruigrok $Y M$, von Und Zu Fraunberg $M$, van Duijn $C M$, et al. : Susceptibility loci for intracranial aneurysm in European and Japanese populations. Nat Genet 40 : 1472-1477, 2008

3. Caranci F, Briganti F, Cirillo L, Leonardi M, Muto M : Epidemiology and genetics of intracranial aneurysms. Eur J Radiol 82 : 1598-1605, 2013

4. Chalouhi N, Ali MS, Jabbour PM, Tjoumakaris SI, Gonzalez LF, Rosenwasser RH, et al. : Biology of intracranial aneurysms: role of inflammation. J Cereb Blood Flow Metab 32 : 1659-1676, 2012

5. Cho YD, Jeon JP, Rhim JK, Park JJ, Yoo RE, Kang HS, et al. : Progressive thrombosis of small saccular aneurysms filled with contrast immediately after coil embolization: analysis of related factors and long-term followup. Neuroradiology 57 : 615-623, 2015

6. Cho YD, Kim SE, Lim JW, Choi HJ, Cho YJ, Jeon JP : Protected versus unprotected carotid artery stenting : meta-analysis of the current literature. J Korean Neurosurg Soc 61 : 458-466, 2018

7. Du Y, Martin JS, McGee J, Yang Y, Liu EY, Sun Y, et al. : A SNP panel and online tool for checking genotype concordance through comparing QR codes. PLoS One 12 : e0182438, 2017

8. Fuying Z, Yingying Y, Shining Z, Kezhong Z, Yanyan S, Xuemei Z, et al. : Novel susceptibility genes were found in a targeted sequencing of stroke patients with or without depression in the Chinese Han population. J Affect Disord 255 : 1-9, 2019

9. Hashikata H, Liu W, Inoue K, Mineharu Y, Yamada S, Nanayakkara S, et al. : Confirmation of an association of single-nucleotide polymorphism rs1333040 on 9p21 with familial and sporadic intracranial aneurysms in Japanese patients. Stroke 41 : 1138-1144, 2010

10. Hixson JE, Jun G, Shimmin LC, Wang Y, Yu G, Mao C, et al. : Whole exome sequencing to identify genetic variants associated with raised atherosclerotic lesions in young persons. Sci Rep 7 : 4091, 2017

11. Hong EP, Jeon JP, Kim SE, Yang JS, Choi HJ, Kang SH, et al. : A novel association between lysyl oxidase gene polymorphism and intracranial aneurysm in Koreans. Yonsei Med J 58 : 1006-1011, 2017

12. Hong EP, Kim BJ, Cho SS, Yang JS, Choi HJ, Kang SH, et al. : Genomic variations in susceptibility to intracranial aneurysm in the Korean population. J Clin Med 8 : 275, 2019

13. Hong EP, Kim BJ, Kim C, Choi HJ, Jeon JP : Association of sox17 gene polymorphisms and intracranial aneurysm: a case-control study and meta-analysis. World Neurosurg 110 : e823-e829, 2018

14. Hong EP, Park JW : Sample size and statistical power calculation in genetic association studies. Genomics Inform 10 : 117-122, 2012

15. Jeon JP, Cho YD, Rhim JK, Yoo DH, Kang HS, Kim JE, et al. : Extended monitoring of coiled aneurysms completely occluded at 6-month followup: late recanalization rate and related risk factors. Eur Radiol 26 : 3319-3326, 2016

16. Jeon JP, Hong EP, Kim JE, Ha EJ, Cho WS, Son YJ, et al. : Genetic risk assessment of elastin gene polymorphisms with intracranial aneurysm in Koreans. Neurol Med Chir (Tokyo) 58 : 17-22, 2018

17. Kim BJ, Kim Y, Hong EP, Jeon JP, Yang JS, Choi HJ, et al. : Correlation between altered DNA methylation of intergenic regions of ITPR3 and development of delayed cerebral ischemia in subarachnoid hemorrhage patients. World Neurosurg 130 : e449-e456, 2019

18. Kim CH, Jeon JP, Kim SE, Choi HJ, Cho YJ : Endovascular treatment with intravenous thrombolysis versus endovascular treatment alone for acute anterior circulation stroke : a meta-analysis of observational studies. J Korean Neurosurg Soc 61 : 467-473, 2018

19. Kim TG, Kim NK, Baek MJ, Huh R, Chung SS, Choi JU, et al. : The rela- 
tionships between endothelial nitric oxide synthase polymorphisms and the formation of intracranial aneurysms in the Korean population. Neurosurg Focus 30 : E23, 2011

20. Lee $S$, Kim IK, Ahn JS, Woo DC, Kim ST, Song S, et al. : Deficiency of endothelium-specific transcription factor Sox17 induces intracranial aneurysm. Circulation 131 : 995-1005, 2015

21. Li B, Hu C, Liu J, Liao X, Xun J, Xiao M, et al. : Associations among genetic variants and intracranial aneurysm in a Chinese population. Yonsei Med J 60 : 651-658, 2019

22. Nahed BV, Bydon M, Ozturk AK, Bilguvar K, Bayrakli F, Gunel M : Genetics of intracranial aneurysms. Neurosurgery 60 : 213-225; discussion 225-226, 2007

23. Petersen BS, Fredrich B, Hoeppner MP, Ellinghaus D, Franke A : Opportunities and challenges of whole-genome and -exome sequencing. BMC Genet $18: 14,2017$
24. Song MK, Kim MK, Kim TS, Joo SP, Park MS, Kim BC, et al. : Endothelial nitric oxide gene T-786C polymorphism and subarachnoid hemorrhage in Korean population. J Korean Med Sci 21 : 922-926, 2006

25. Teer JK, Mullikin JC : Exome sequencing: the sweet spot before whole genomes. Hum Mol Genet 19(R2) : R145-R151, 2010

26. Wu Y, Li Z, Shi Y, Chen L, Tan H, Wang Z, et al. : Exome sequencing identifies LOXL2 mutation as a cause of familial intracranial aneurysm. World Neurosurg 109 : e812-e818, 2018

27. Yamada Y, Kato K, Oguri M, Horibe H, Fujimaki T, Yasukochi Y, et al. : Identification of nine genes as novel susceptibility loci for early-onset ischemic stroke, intracerebral hemorrhage, or subarachnoid hemorrhage. Biomed Rep 9 : 8-20, 2018

28. Yasuno K, Bilguvar K, Bijlenga P, Low SK, Krischek B, Auburger G, et al. : Genome-wide association study of intracranial aneurysm identifies three new risk loci. Nat Genet 42 : 420-425, 2010 